\title{
Altered Reactivity of Tertiary Mesenteric Arteries following Acute Myocardial Ischemia
}

\author{
Young Soo Han Frank V. Brozovich \\ Division of Cardiovascular Diseases, Mayo Clinic, Rochester, Minn., USA
}

\section{Key Words}

Mesenteric artery $\cdot$ Acetylcholine $\cdot 8$-Bromo-cGMP $\cdot$ Myosin phosphatase targeting subunit $1 \cdot$ Nonmuscle myosin

\begin{abstract}
Background: It is unknown if cardiac ischemia has any deleterious effect on the contractile properties of nonischemic, peripheral vascular beds. Thus, the objective of the present study was to determine whether acute myocardial ischemia results in peripheral vascular dysfunction. Methods and Results: This study characterized force maintenance and the sensitivity to acetylcholine (ACh)-mediated smooth muscle (SM) relaxation of tertiary (3rd) mesenteric arteries from Sprague-Dawley rats following $30 \mathrm{~min}$ of myocardial ischemia. Both the phosphorylation of nonmuscle (NM) light chain (LC) and SM-LCs as well as the expression of myosin phosphatase targeting subunit 1 (MYPT1) were also determined. Our data demonstrate that acute myocardial ischemia resulted in vascular dysfunction of 3 rd mesenteric vessels, characterized by decreases in force maintenance, AChand CGMP-mediated SM relaxation, the phosphorylation of NM-LCs and SM-LCs, and MYPT1 expression. Ischemia was also associated with an increase in protein polyubiquitination, suggesting that during ischemia MYPT1 is targeted for degradation or proteolysis. Conclusion: Acute myocardial
\end{abstract}

ischemia produces peripheral vascular dysfunction; the changes in LC phosphorylation and MYPT1 expression result in a decrease in both tone and the sensitivity to NO-mediated SM relaxation of the peripheral vasculature.

Copyright $\odot 2012$ S. Karger AG, Basel

\section{Introduction}

The effects of acute ischemia on the ischemic vascular bed include decreases in vascular tone and the sensitivity of smooth muscle (SM) to nitric oxide (NO)-mediated vasodilatation $[1,2]$. These changes in vascular reactivity occur within $30 \mathrm{~min}$ of ischemia and are thought to be mediated by an increase in the production of free radicals as well as interleukin-1 and tumor necrosis factor- $\alpha$. Recurrent ischemia often leads to heart failure, which has been documented to be associated with an increase in peripheral vascular tone as well as a decrease in the sensitivity to NO-mediated vasodilatation [1-4]. However, the effect of acute myocardial ischemia on the vasoreactivity of nonischemic peripheral vascular SM has not been investigated.

Contraction of SM is dependent on the level of phosphorylation of the $20-\mathrm{kDa}$ regulatory SM myosin light chain (SM-LC), which is determined by the relative ac-

\section{KARGER \\ Fax +4161306 1234 \\ E-Mail karger@karger.ch}

www.karger.com
(C) 2012 S. Karger AG, Basel

$1018-1172 / 13 / 0502-0100 \$ 38.00 / 0$

Accessible online at:

www.karger.com/jvr
Dr. Frank V. Brozovich

Division of Cardiovascular Diseases, Mayo Clinic

200 First Street SW

Rochester, MN 55905 (USA)

E-Mail brozovich.frank@mayo.edu 
tivities of myosin LC (MLC) kinase and MLC phosphatase [5-7]. However, recent evidence suggests that nonmuscle (NM) myosin also participates in the force maintenance phase of SM contraction and hence the regulation of vascular tone [5-8]. In addition, NO-mediated vasodilatation is considered a fundamental response of the vasculature [9], and changes in the sensitivity of NO-mediated SM relaxation are produced, in part, by an alteration in the expression of myosin phosphatase targeting subunit 1 (MYPT1) of MLC phosphatase [10, 11], specifically by a decrease in the expression of the leucine zipper-positive (LZ+) MYPT1 isoform [11-13].

This study was designed to determine the effect of 30 min of myocardial ischemia on the reactivity of tertiary (3rd) mesenteric arteries. Our data suggest that acute ischemia causes a dysregulation of SM and NM myosin, which results in a decrease in vascular tone and a reduction in the expression of MYPT1, which attenuates the sensitivity of NO-mediated vasodilatation.

\section{Materials and Methods}

Rat Acute Ischemia Model and Mesenteric Artery Preparation The Institutional Animal Care and Use Committee of the Mayo Clinic approved all experimental protocols and animal care, and the study conforms to the guidelines of the National Institutes of Health. Our model of cardiac ischemia has been described in detail previously [14-16]; briefly, adult male SpragueDawley rats (250-350 g) were injected intramuscularly with ketamine $(60 \mathrm{mg} / \mathrm{kg})$ and xylazine $(12 \mathrm{mg} / \mathrm{kg})$ and ventilated with room air. Animals were closely monitored for pain by testing the blink reflex. The heart was exposed by a midline thoracotomy, and a ligature was placed near the bifurcation of the left coronary artery, restricting flow through the left anterior descending and circumflex artery. Animals were divided into two experimental groups, i.e. one group with $30 \mathrm{~min}$ of perfusion (sham) and the other with $30 \mathrm{~min}$ of coronary occlusion (ischemia). Following either $30 \mathrm{~min}$ of perfusion or ischemia, mesenteric vessels were exteriorized, and mesenteric arteries were gently excised and transferred to a vessel chamber containing $\mathrm{Ca}^{2+}$-free Tyrode's solution (in mM: $135 \mathrm{NaCl}, 4 \mathrm{KCl}, 1 \mathrm{MgCl}_{2}, 0.33 \mathrm{Na}_{2} \mathrm{HPO}_{4}, 0.03$ EDTA, 10 glucose, 10 HEPES; pH 7.4). Following careful removal of connective and adipose tissue, 3rd branches of the mesenteric artery were isolated.

\section{Mechanical Studies}

For force recordings, isolated 3rd mesenteric preparations (100-200 $\mu \mathrm{m}$ in diameter, approx. $2 \mathrm{~mm}$ in length) with an intact endothelium were mounted using wires ( $40 \mu \mathrm{m}$ in diameter) on a DMT (Mulvany) 4-channel myograph system [17-19] and stretched to $L_{o}$ (the length for maximal force) in the myograph chamber containing continuously oxygenated physiological saline solution (in mM: $140 \mathrm{NaCl}, 3.7 \mathrm{KCl}, 2.5 \mathrm{CaCl}_{2}, 0.81 \mathrm{MgSO}_{4}$, $1.19 \mathrm{KH}_{2} \mathrm{PO}_{4}, 0.03$ EDTA, 5.5 glucose, 25 HEPES; $\left.\mathrm{pH} 7.4\right)$. The muscle preparations were stimulated to contract with $80 \mathrm{mM}$
$\mathrm{KCl}$ depolarization (in mM: $64.5 \mathrm{NaCl}, 80 \mathrm{KCl}, 2.5 \mathrm{CaCl}_{2}, 0.81$ $\mathrm{MgSO}_{4}, 1.19 \mathrm{KH}_{2} \mathrm{PO}_{4}, 0.03$ EDTA, 5.5 glucose, 25 HEPES; $\mathrm{pH}$ 7.4) or $10 \mu \mathrm{M}$ phenylephrine (PE). For one group of vessels, the force response to either $\mathrm{KCl}$ depolarization or $\mathrm{PE}$ was recorded for 10-15 $\mathrm{min}$, before the vessel was relaxed. In a separate series of experiments, the vessels were depolarized, and after the force reached a steady state, the dose-response relationship of force relaxation produced by either acetylcholine (ACh) or the cellpermeable cGMP analog 8-bromo-cGMP (8-Br-cGMP) was determined.

\section{SM and NM Myosin LC Phosphorylation}

The time course of SM-LC and NM-LC phosphorylation in perfused versus ischemic mesenteric preparations was determined using 2-dimensional (2-D) gel electrophoresis [20]. We have demonstrated that this technique resolves the phosphorylated and nonphosphorylated NM-LCs and the phosphorylated and nonphosphorylated SM-LCs as four distinct spots [20]. The phosphorylation of NM-LC and SM-LC can then be determined using densitometry [20]. For these experiments, the vessels were activated with $80 \mathrm{mM} \mathrm{KCl}$ depolarization, and LC phosphorylation was determined at 0 (rest), 2 and $10 \mathrm{~min}$ of $\mathrm{KCl}$ depolarization. At the specified time, mesenteric microvessels were frozen in liquid nitrogen. As previously described $[15,20]$, frozen tissues were homogenized in 2-D gel extraction buffer [7 $\mathrm{M}$ urea, $2 \mathrm{M}$ thiourea, 4\% CHAPS, 1\% 3-5.6 immobilized pH gradient (IPG) buffer] and EDTA-free Complete Protease Inhibitor (Roche, Indianapolis, Ind., USA). The homogenates were cleared of lipids and extraneous salts using the 2-D gel clean-up kit (Amersham Biosciences, Piscataway, N.J., USA). The acidic halves of 13-cm IPG DryStrip gels ( $\mathrm{pH}$ 3-5.6 nonlinear gradient) were rehydrated in the presence of suitable amounts of sample in rehydration buffer solution (7 M urea, $2 \mathrm{M}$ thiourea, 2\% CHAPS, 0.5\% pH 3.5-5 IPG buffer, $0.002 \%$ bromophenol blue and $12 \mu \mathrm{l} / \mathrm{ml}$ Destreak Reagent) for at least $10 \mathrm{~h}$ in the 'face-down' mode on the Ettan IPG rehydration tray and then resolved by isoelectric focusing in the 'face-up' mode on an Ettan IPGphor III (GE Healthcare). Following isoelectric focusing, the gel strips were equilibrated in $6 \mathrm{M}$ urea, 50 $\mathrm{mM}$ Tris-HCL, pH 6.4, 30\% glycerol, $2 \%$ (w/v) SDS and $0.002 \%$ bromophenol blue, first containing $130 \mathrm{~mm}$ DTT for $15 \mathrm{~min}$ and then containing $135 \mathrm{~mm}$ iodoacetamide for 15 min before undergoing SDS-PAGE for protein separation by molecular weight. Subsequently, resolved 2-D SDS-PAGE gels were silver stained. To measure the extent of SM-LC or NM-LC phosphorylation, stained gels were scanned using a Personal Densitometer SI, and the spots were quantified using ImageQuant TL software. The two spots closest to the anode represent the phosphorylated and nonphosphorylated NM-LC, and the two spots nearest the cathode represent the phosphorylated and nonphosphorylated SM-LC [20]. The NM-LC phosphorylation level is determined by the ratio of phosphorylated NM-LC to total NM-LC, and the SM-LC phosphorylation level is similarly calculated as the ratio of phosphorylated SM-LC to total SM-LC [20].

\section{Immunoblotting}

Western blots were used to determine protein expression in perfused versus ischemic mesenteric preparations as previously described [10, 11, 13, 21]. Briefly, frozen total protein homogenates were suspended in SDS sample buffer. Proteins were resolved by SDS-PAGE using $8 \%$ gels with an acrylamide/bisacrylamide ratio 


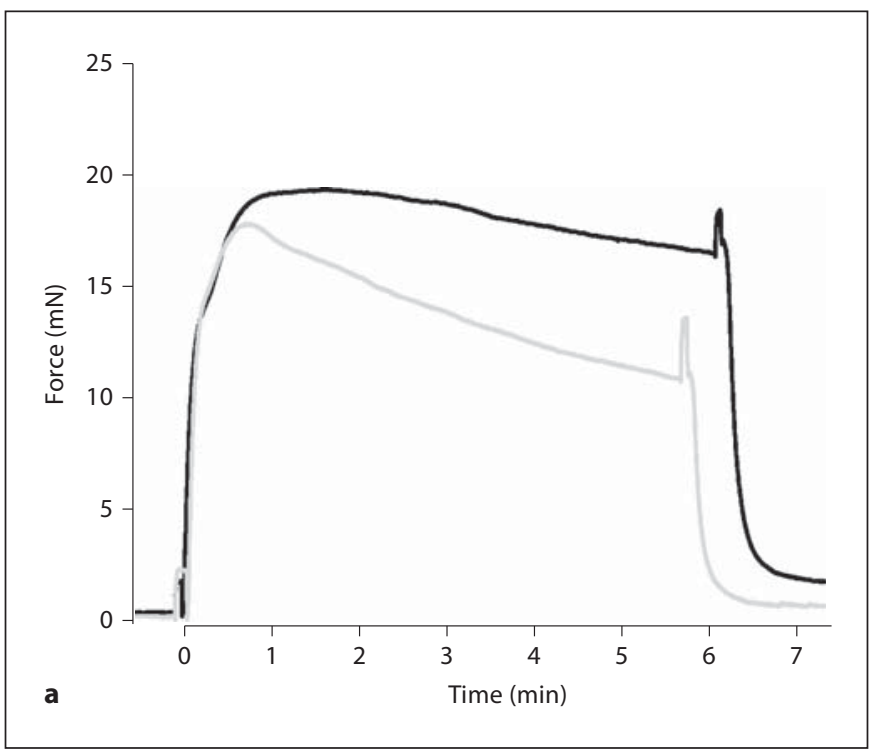

Fig. 1. Ischemia decreases force maintenance. a Representative force tracings of perfused (black line) and ischemic (gray line) mesenteric microvessels during $80 \mathrm{mM} \mathrm{KCl}$ depolarization. Following $\mathrm{KCl}$ depolarization, force rapidly rises to a peak in both perfused and ischemic preparations. However, force maintenance was reduced in the ischemic mesenteric vessels compared to that

of 29:1. Following protein separation by SDS-PAGE, the proteins were transferred onto a polyvinylidene difluoride membrane. To detect MYPT1, LZ+ MYPT1, desmin and actin, a rabbit polyclonal anti-MYPT1 (Upstate Biotechnology), a mouse monoclonal anti-LZ+ MYPT1 isoform [10, 11], a mouse monoclonal antidesmin (D1033, Sigma) and an anti-actin (A2066, Sigma) antibody were used. Following washing, the blots were incubated with Cy3-labeled antimouse IgG (Jackson Immunoresearch) or Cy5labeled antirabbit IgG (GE Life Sciences). Blots were scanned on a Typhoon 9410 imager, and the scanned images were analyzed using ImageQuant TL software. For all sample preparations, the quantified signal for the protein of interest was divided by the $\beta$ actin signal to provide an internally controlled value of the relative expression. To normalize values across different blots, one of the sample preparations was chosen as a standard sample and loaded on all Western blots.

Protein polyubiquitination was assessed using immunoblotting with a mouse monoclonal antibody [Ub (P4D1), Santa Cruz Biotechnology], and ubiquitination was quantified with densitometry and normalized to actin, as described above.

\section{Statistical Analysis}

All data are presented as means \pm SEM, and $n$ represents the number of animals in each group. Student's t test was performed to evaluate significant differences between the two groups, and differences were considered to be significant at $p<0.05$. When multiple comparisons between groups were necessary, a Bonferroni correction was performed.

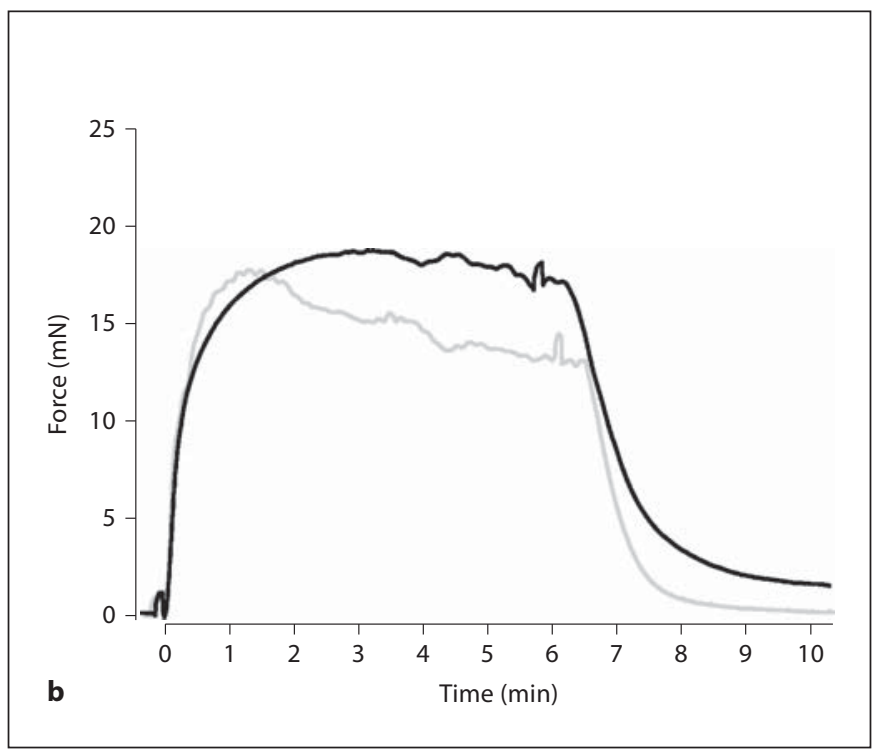

in perfused vessels $(\mathrm{p}<0.05)$. $\mathbf{b}$ Representative force tracings of perfused (black line) and ischemic (gray line) mesenteric microvessels during PE activation. Force rapidly rises to a peak in both perfused and ischemic preparations. However, force maintenance was reduced in the ischemic mesenteric vessels compared to that in perfused vessels $(\mathrm{p}<0.05)$.

\section{Results}

Following $\mathrm{KCl}$ depolarization, force rapidly rose to a peak in both perfused and ischemic preparations (16.8 \pm 2.2 vs. $16.3 \pm 2.1 \mathrm{mN} ; \mathrm{n}=6 ; \mathrm{p}>0.05)$. In perfused preparations, force slowly declined to $78.6 \pm 3.1 \%(n=6)$ of the peak force during force maintenance (fig. 1a). In contrast, for the ischemic 3rd mesenteric arterial vessels, the ability to maintain force was significantly compromised (fig. 1a); force maintenance was $70.6 \pm 1.1 \%$ of peak force $(n=6 ; p<0.05$ vs. perfused). With PE stimulation, force also rose to a peak in perfused and ischemic preparations $[13.8 \pm 1.1 \mathrm{mN}(\mathrm{n}=5)$ vs. $12.5 \pm 1.5 \mathrm{mN}(\mathrm{n}=4) ; \mathrm{p}>$ 0.05]. In perfused preparations, force remained at a steady state $(97.8 \pm 1.2 \%$ of peak; $\mathrm{p}>0.05)$, while in ischemic preparations, force fell by approximately $30 \%$ to $72.3 \pm$ $6.5 \%$ of peak force $(\mathrm{p}<0.05)$ during force maintenance (fig. 1b).

The expression of NM myosin was similar in perfused and ischemic $3 \mathrm{rd}$ mesenteric vessels $(7.8 \pm 1.9$ vs. $10.8 \pm$ $2.4 \% ; \mathrm{n}=6 ; \mathrm{p}>0.05)$. Thus, to explore the mechanism underlying the ischemia-induced decline in force maintenance, we determined the time course of SM-LC and NM-LC phosphorylation. For depolarization of perfused 


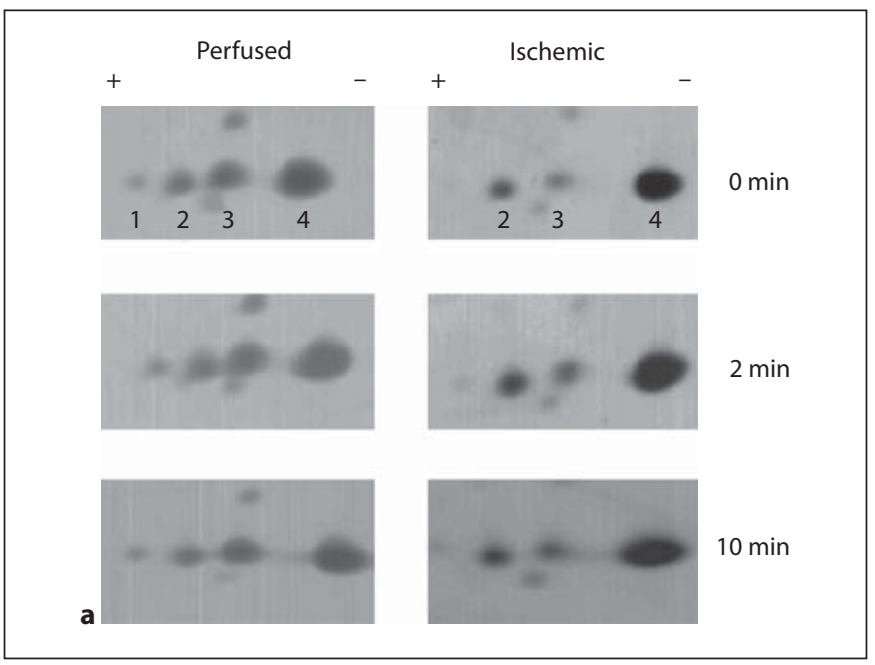

Fig. 2. SM-LC and NM-LC phosphorylation in ischemic and perfused vessels. a Representative silver-stained 2-D gel of rat mesenteric artery tissue lysates. Spots 1 and 2 represent phosphorylated and nonphosphorylated NM-LC, respectively, while spots 3 and 4 represent phosphorylated and nonphosphorylated SM-LC. NM-LC phosphorylation is computed as the density of $[1 /(1+2)]$ $\times 100 \%$, and SM-LC phosphorylation is $[3 /(3+4)] \times 100 \%$, while the expression of NM myosin is calculated as $[(1+2) /(1+2+3+$ 4) $\times 100 \%$. b, c Time course of SM-LC (b) and NM-LC (c) phosphorylation in perfused and ischemic 3rd mesenteric vessels during $\mathrm{KCl}$ depolarization. ${ }^{*} \mathrm{p}<0.05$ versus LC phosphorylation at $0 \min (n=5)$.

preparations, SM-LC phosphorylation did not change, but there was a significant increase in NM-LC phosphorylation $(7.8 \pm 1.9 \%$ at rest vs. $16.6 \pm 3.5 \%$ at $2 \mathrm{~min} ; \mathrm{p}<$ $0.05 ; \mathrm{n}=5$; fig. 2 ), suggesting that in $3 \mathrm{rd}$ mesenteric vessels both force activation and maintenance are regulated by the activation of NM myosin. For depolarization of ischemic preparations, there was no detectable NM-LC phosphorylation. Resting SM-LC phosphorylation compared to perfused was significantly lower, and depolarization resulted in a significant increase in SM-LC phosphorylation $(6.7 \pm 1.4 \%$ at rest vs. $12.5 \pm 1.7 \%$ at $2 \mathrm{~min}$; $\mathrm{p}<0.05 ; \mathrm{n}=5$; fig. 2). These data suggest that following myocardial ischemia, force activation and maintenance are dependent on the activation of SM myosin, and ischemia results in a change in the activation (phosphorylation) of both SM and NM myosin.
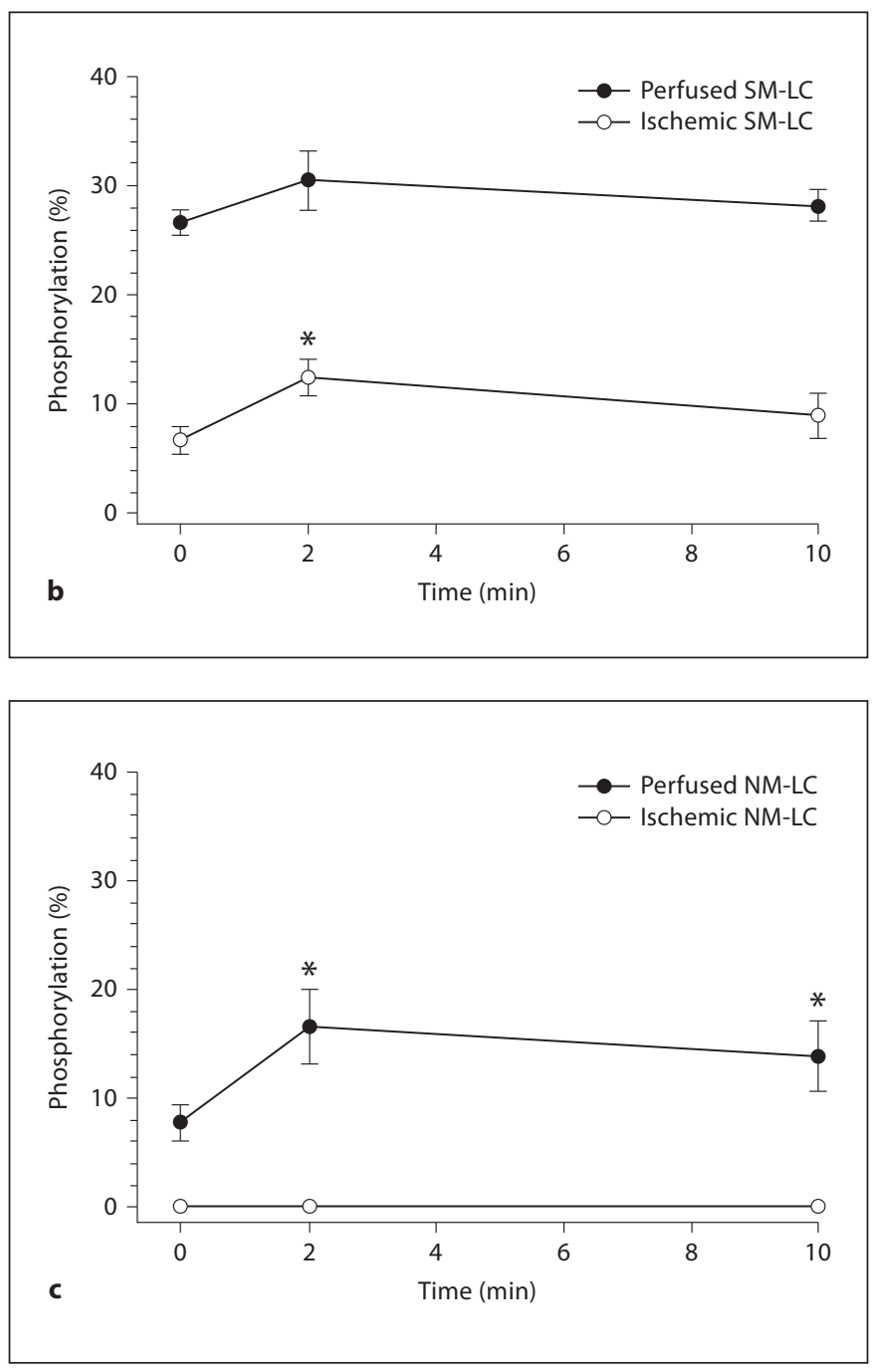

We investigated NO-mediated signaling by determining the dose-response relationship of ACh-induced relaxation of 3rd mesenteric arteries. For the perfused and ischemic preparations, ACh produced a dose-dependent relaxation (fig. 3a). For perfused versus ischemic vessels, there was no significant difference in the sensitivity to ACh (median effective dose $100 \pm 8$ vs. $97 \pm 4 \mathrm{nM}$; $>$ $0.05 ; n=5)$. However, ACh produced a significantly larger maximal relaxation in the perfused 3rd mesenteric vessels ( $56 \pm 6$ vs. $38 \pm 4 \% ; \mathrm{p}<0.05 ; \mathrm{n}=5$; fig. $3 \mathrm{a}$ ). To determine whether the impairment of ACh-mediated SM relaxation in ischemic mesenteric vessels was due to a defect at the level of the SM contractile apparatus, similar experiments were performed using 8-Br-cGMP (fig. 3b); similar to ACh-mediated relaxation, there was no difference in the sensitivity to 8 -Br-cGMP (median effective 
Fig. 3. Ischemia decreases $\mathrm{ACh}$ - and 8-BrcGMP-mediated SM relaxation. a The dose-response relationship of SM relaxation produced by ACh. The magnitude of relaxation produced by $\mathrm{ACh}$ is blunted $\left({ }^{*} \mathrm{p}<0.05 ; \mathrm{n}=5\right)$ in the 3 rd mesenteric vessels from ischemic compared to perfused preparations. b The dose-response relationship of SM relaxation produced by 8-Br-cGMP. The magnitude of relaxation produced by 8 -Br-cGMP is blunted $\left({ }^{*} \mathrm{p}<\right.$ $0.05 ; \mathrm{n}=5)$ in the $3 \mathrm{rd}$ mesenteric vessels from ischemic compared to perfused preparations.
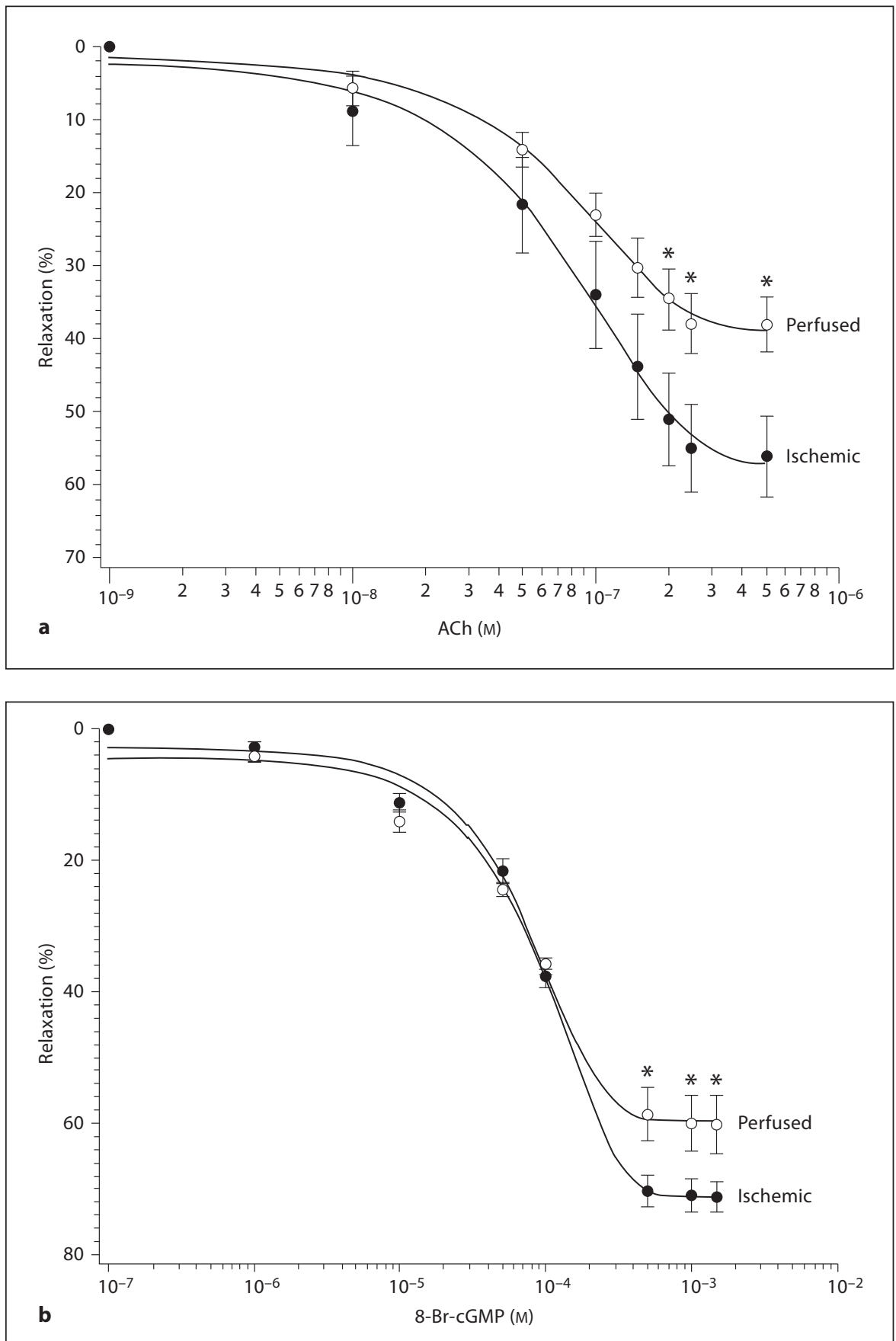

dose $96 \pm 4$ vs. $77 \pm 11 \mu \mathrm{M} ; \mathrm{p}>0.05 ; \mathrm{n}=5$ ), but the maximum relaxation was significantly reduced in the ischemic as compared to the perfused preparations (60 \pm 4 vs. $71 \pm 2 \% ; \mathrm{p}<0.05 ; \mathrm{n}=5$ ).

We have previously demonstrated that a decrease in the sensitivity to cGMP-mediated SM relaxation is produced by a decrease in the expression of the LZ+ MYPT1 isoform $[10,11]$. Thus, Western blots were used to evaluate MYPT1 and LZ+ MYPT1 expression in perfused and ischemic mesenteric vessels (fig. 4), and the expression of both MYPT1 and LZ+ MYPT1 in ischemic mesenteric vessels was significantly depressed when compared to perfused vessels (MYPT1: $64 \pm 8 \%, \mathrm{p}<0.05, \mathrm{n}=4$; LZ+ MYPT1: $74 \pm 6 \%, \mathrm{p}<0.05, \mathrm{n}=4$; fig. 4). 
We also examined Coomassie staining of SDS gels and noted that there was a significant difference in the density of two protein bands comparing perfused and ischemic vessels (fig. 5), at approximately $25 \mathrm{kDa}$ and approximately $60 \mathrm{kDa}$. We identified these proteins using mass spectrometry; the $25-\mathrm{kDa}$ protein is $\mathrm{SM}-22 \alpha$, while the $60-\mathrm{kDa}$ protein(s) is either ATP synthase, adenylyl cyclase, glutamate dehydrogenase and/or desmin. The expression of SM-22 $\alpha$ decreased during depolarization (66 $\pm 3 \%$ of perfused at $10 \mathrm{~min} ; \mathrm{p}<0.05 ; \mathrm{n}=4)$. Desmin expression was similar in perfused and ischemic vessels (online suppl. fig. S1; for all online suppl. material, see www.karger.com/doi/10.1159/000343015), and thus, the identity and significance of the protein(s) at approximately $60 \mathrm{kDa}$ with lower expression in the ischemic preparations will require further study.

To investigate if the decline in protein expression could be due to degradation by a ubiquitin-proteasome system, we examined the extent of protein polyubiquitination with immunoblotting. A significant increase in protein polyubiquitination was observed in the ischemic compared to perfused mesenteric vessels $(\mathrm{p}<0.05$; online suppl. fig. S2).

\section{Discussion}

The effect of acute myocardial ischemia on the vasoreactivity of the coronary vessels has been documented $[1,2]$, but the effect of myocardial ischemia on the reactivity of noncardiac resistance microvessels has not been investigated. Therefore, the main objective of this study was to investigate the effect of acute ischemia on the vascular function of nonischemic resistance vessels. In the present study, we found that acute myocardial ischemia produced dysfunction in 3rd mesenteric vessels, which was characterized by a decrease in force maintenance for both $\mathrm{KCl}$ and PE stimulation and also a decrease in NO-mediated SM relaxation.

\section{Force Maintenance}

Recent evidence suggests that NM myosin participates in the force maintenance phase of SM contraction and the regulation of vascular tone [20,22-25]; we have reported that a $50 \%$ reduction in the expression of NM myosin in the rat aorta was associated with a $25 \%$ decrease in force maintenance [20]. In 3rd mesenteric vessels, NM myosin expression is approximately $10 \%$ of total myosin heavy chain, similar to NM myosin expression in rat aorta [20]. In perfused preparations, $\mathrm{KCl}$ depolarization resulted in

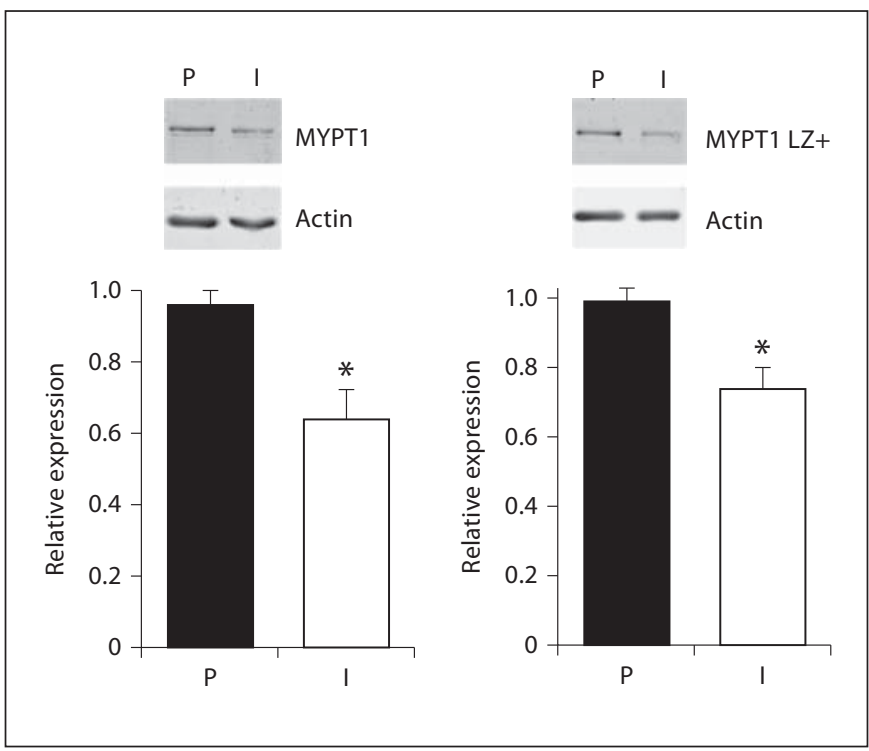

Fig. 4. MYPT1 and LZ+ MYPT1 expression. Western blots of MYPT1 and LZ+ MYPT1 expression in perfused (P) versus ischemic (I) preparations. The bar graphs demonstrate that MYPT1 and LZ+ MYPT1 expression was significantly reduced $\left({ }^{*} \mathrm{p}<0.05\right.$; $\mathrm{n}=4$ ) in ischemic compared to perfused 3rd mesenteric vessels.

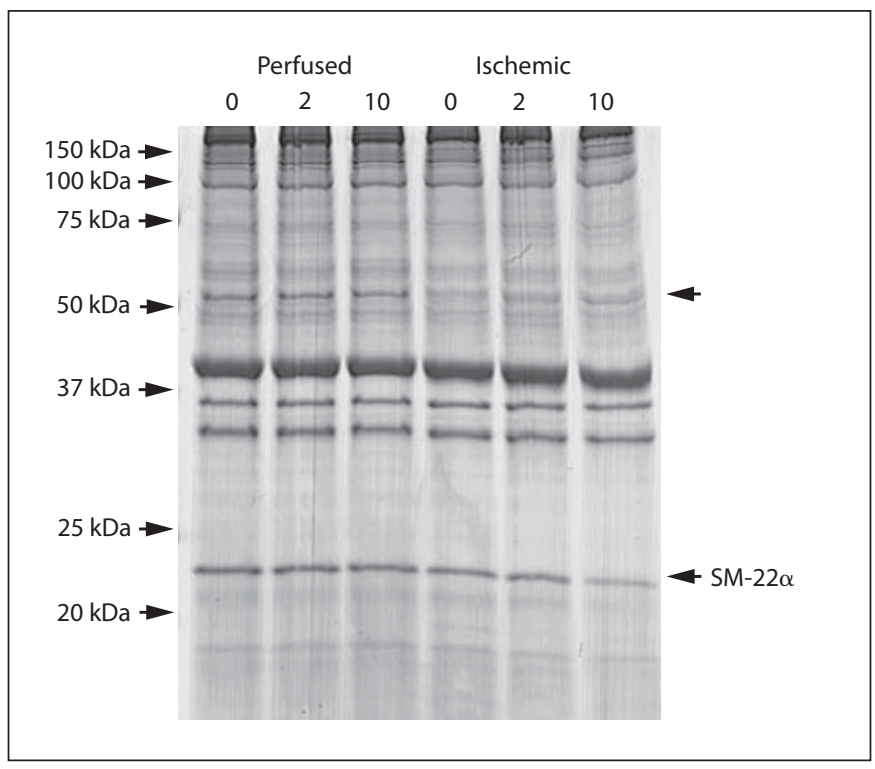

Fig. 5. Ischemia decreases protein expression in ischemic mesenteric vessels. Representative Coomassie-stained SDS-PAGE of tissue lysates during $\mathrm{KCl}$ depolarization (0, 2 and $10 \mathrm{~min}$ ). The expression of SM-22 $\alpha$ decreased during depolarization $(\mathrm{p}<0.05$; $n=4)$, while the expression of the protein(s) representing the band at $60 \mathrm{kDa}$ (upper arrow) was lower in the ischemic vessels $(\mathrm{p}<$ $0.05 ; \mathrm{n}=4)$. 
a sustained increase in phosphorylation of NM-LC but not of SM-LC (fig. 2). These data suggest that NM myosin participates in the regulation of force and vascular tone in 3rd mesenteric vessels. Thus, a change in the phosphorylation of NM myosin in ischemic vessels would be expected to decrease force maintenance. Following $30 \mathrm{~min}$ of myocardial ischemia, there was no detectable phosphorylation of NM-LC. However, $\mathrm{KCl}$ depolarization produced a significant increase in SM-LC phosphorylation (fig. 2). These results demonstrate that ischemia changes the mechanism for force regulation in 3rd mesenteric arteries; ischemia produces a shift from a dependence on the activation of NM myosin to SM myosin. Thus, as proposed by others $[8,26,27]$, our data demonstrate that the activation of NM myosin is important for force maintenance.

The magnitude of the change in the phosphorylation of the LCs in the mesenteric vessels was approximately 6-8\% (fig. 2). In resistance vessels, pressurization has been demonstrated to be important for the absolute magnitude of LC phosphorylation [28], and in the present study, it is possible that the changes in both NM-LC and SM-LC phosphorylation would have been larger if the vessels had been pressurized. Further, following depolarization, force peaks at 30-60 s, which would suggest that LC phosphorylation would be at a maximum earlier than the 2 -min time point measured in our study. However, force activation during PE stimulation and depolarization of rabbit urethral SM is regulated by a Rho kinase-dependent pathway, but with no detectable change in LC phosphorylation [29]. The NM-LC and SM-LC differ by only 9 amino acids, and the anti-SM-LC antibody detects both SM-LC and NM-LC [20]. A Rho kinase-mediated pathway has been demonstrated to activate NM myosin [20], and thus for urethral SM, an increase in NM-LC phosphorylation may have been detected using 2-D SDS-PAGE.

\section{NO-Mediated SM Relaxation}

Others have demonstrated that ischemia produces endothelial dysfunction of the ischemic vascular bed $[1,2]$. NO signaling causes SM relaxation using both $\mathrm{Ca}^{2+}$-dependent and $\mathrm{Ca}^{2+}$-independent $\left(\mathrm{Ca}^{2+}\right.$ desensitization) mechanisms [30-32]. During NO stimulation, NO diffuses into the SM cell to activate guanylate cyclase, which increases cGMP, subsequently activating protein kinase G. Protein kinase $G$ mediates phosphorylation of a number of targets to produce a reduction in intracellular $\mathrm{Ca}^{2+}$, leading to a $\mathrm{Ca}^{2+}$-dependent relaxation, but it also activates MLC phosphatase to produce $\mathrm{Ca}^{2+}$ desensitization. Our data demonstrate that myocardial ischemia produces a decrease in the relaxation of 3rd mesenteric vessels produced by both ACh and 8-Br-cGMP (fig. 3). We have demonstrated that a decrease in LZ+ MYPT1 expression produces a reduction in cGMP-mediated SM relaxation $[10,11]$, and a decrease in both LZ+ MYPT1 expression and cGMP-mediated SM relaxation has been documented in nitrate tolerance $[33,34]$ and heart failure $[12,35]$ as well as other vascular diseases [36]. Similarly, our data demonstrate that myocardial ischemia produces a decrease in the expression of both MYPT1 and the LZ+ MYPT1 isoform in 3rd mesenteric vessels (fig. 4). Although myocardial ischemia could produce endothelial dysfunction and a decrease in NO, these data demonstrate a defect at the level of the SM specifically; the decrease in MYPT1 expression contributes to the molecular mechanism responsible for the reduction in the relaxation of the mesenteric vessels by NO-based vasodilators.

The ischemia-induced decrease in MYPT1 expression (fig. 4) could suggest that acute ischemia triggers calpaininduced proteolysis or ubiquitin-proteasome-based degradation. During depolarization with $80 \mathrm{mM} \mathrm{KCl}$, the intracellular $\mathrm{Ca}^{2+}$ concentration increases to a sustained steady state [37], which could suggest that the decrease in MYPT1 expression is due to a $\mathrm{Ca}^{2+}$-dependent process, such as calpain. However, calpain degradation produces large fragments of the intact protein [38], which we did not observe (fig. 5). Ubiquitin covalently attaches to proteins that are subsequently delivered to the proteasome for rapid degradation. The $26 \mathrm{~S}$ proteasome is responsible for the degradation of polyubiquitinated proteins [39] and is expressed in vascular SM $[40,41]$. The $26 \mathrm{~S}$ proteasome is composed of a $20 \mathrm{~S}$ catalytic core and two $19 \mathrm{~S}$ regulatory particles [39]. The $20 \mathrm{~S}$ core is composed of $2 \alpha$ rings $(\alpha 1-$ 7 ) and $2 \beta$ rings ( $\beta 1-7)$; the $\alpha$ rings bind proteasome regulators and identify ubiquitinated proteins targeted for degradation $[39,42]$, and the $\beta$ rings contain catalytically active sites $[39,42]$. Thus, an increase in polyubiquitinated protein complexes (online suppl. fig. S2) could suggest that the decrease in protein expression is due to protein degradation by the ubiquitin-proteasome pathway.

In addition to MYPT1, the expression of SM-22 $\alpha$ was lower in the ischemic preparations (fig. 5). SM-22 $\alpha$ [43] is abundant in SM; SM-22 $\alpha$ is a cytoskeletal protein that binds to actin filaments at a ratio of 1:6 actin monomers and has been suggested to regulate the organization of thin filaments within the cytoskeleton of the SM cell [44]. SM$22 \alpha$-deficient mice have no phenotype, although SM tissue mechanics have not been reported [43]. Ali et al. [45] have suggested that both actin and myosin filaments reorganize (shorten) in response to changes in SM length. Thus, one possible explanation for the decrease in SM-22 $\alpha$ in the 
ischemic preparations is that depolarization of the ischemic mesenteric vessel results in an actin filament depolymerization/repolymerization, which shortens the overall thin filament length, resulting in free, unbound SM-22 $\alpha$ that is subsequently targeted for degradation or proteolysis.

The mechanism and/or signaling responsible for triggering the changes in remote, nonischemic vascular beds are unclear. Although we did not measure hemodynamics during the coronary ligation, studies establishing these parameters during acute coronary ischemia have been published for animals [46, 47] and humans [48]. These investigators have demonstrated that there is no significant change in either blood pressure or heart rate during $30 \mathrm{~min}$ of coronary artery ligation in dogs [46] or rats [47]. It is unlikely that production of free radicals in the coronary beds would result in a significant elevation in 3rd mesenteric arteries. The decrease in cardiac output during $30 \mathrm{~min}$ of ischemia could decrease the partial pressure of oxygen and increase the partial pressure of carbon dioxide in the mesenteric beds, which could increase the production of free radicals. However, we would expect free radical modification to be more widespread rather than limited to only MYPT1 and SM-22 $\alpha$. Further, changes in MYPT1 isoform expression have also been documented during development $[49,50]$, as well as in animal models of portal hypertension [51] and pregnancy-induced hypertension [52]; these investigators [51, 52] suggested that changes in pressure and flow regulate MYPT1 isoform expression. Nonetheless, the trigger for the changes in protein expression during ischemia will require further investigation.

\section{Conclusion}

Our data demonstrate that acute myocardial ischemia decreases force maintenance in 3 rd mesenteric vessels stimulated to contract with either $\mathrm{KCl}$, which leads to an increase in intracellular $\mathrm{Ca}^{2+}$, or $\mathrm{PE}$, which increases $\mathrm{Ca}^{2+}$ and also activates signaling pathways for $\mathrm{Ca}^{2+}$ sensitization. Further, acute myocardial ischemia (1) changes the regulation of force from requiring the activation of $\mathrm{NM}$ myosin to activation of SM myosin, which results in an impairment in force maintenance, and (2) decreases the expression of both MYPT1 and the LZ+ MYPT1 isoform, which produces a reduction in the relaxation of 3rd mesenteric vessels in response to NO-based vasodilators. These findings demonstrate that even acute myocardial dysfunction results in vascular dysfunction and in changes of the reactivity of the peripheral vasculature. Further, our results demonstrate that the activation of NM myosin participates in the regulation of vascular tone, and identifying the mechanism underlying the changes in the activation of NM/SM myosin and the expression of MYPT1 will provide insight into the development of therapeutic strategies for myocardial ischemia.

\section{Acknowledgements}

We would like to thank Dr. Ozgur Ogut for his comments and suggestions. This work was supported by the Mayo Clinic and the National Institutes of Health (T32 HL007111 to Y.S.H.).

\section{References}

$\longrightarrow$ Fagan SC, Hess DC, Hohnadel EJ, Pollock DM, Ergul A: Targets for vascular protection after acute ischemic stroke. Stroke 2004;35: $2220-2225$

$\checkmark 2$ Fernandez N, Martinez MA, Climent B, Garcia-Villalon AL, Monge L, Sanz E: Coronary reactivity to endothelin-1 during partial ischemia and reperfusion in anesthetized goats. Role of nitric oxide and prostanoids. Eur J Pharmacol 2002;457:161-168.

3 Francis GS, Cohn JN: Heart failure: mechanisms of cardiac and vascular dysfunction and the rationale for pharmacologic intervention. FASEB J 1990;4:3068-3075.

4 Negrao CE, Hamilton MA, Fonarow GC, Hage A, Moriguchi JD, Middlekauff HR: Impaired endothelium-mediated vasodilation is not the principal cause of vasoconstriction inheartfailure. Am J Physiol2000;278:H168H174.
5 Gong MC, Cohen P, Kitazawa T, Ikebe M, Masuo M, Somlyo AP: Myosin light chain phosphatase activities and the effects of phosphatase inhibitors in tonic and phasic smooth muscle. J Biol Chem 1992;267: 14662-14668.

6 Ogut O, Brozovich FV: Determinants of the contractile properties in the embryonic chicken gizzard and aorta. Am J Physiol 2000;279:C1722-C1732.

7 Ogut O, Yuen SL, Brozovich FV: Regulation of the smooth muscle contractile phenotype by nonmuscle myosin. J Muscle Res Cell Motil 2007;28:409-414.

8 Ekman M, Fagher K, Wede M, Stakeberg K, Arner A: Decreased phosphatase activity, increased $\mathrm{Ca} 2+$ sensitivity, and myosin light chain phosphorylation in urinary bladder smooth muscle of newborn mice. J Gen Physiol 2005;125:187-196.
9 Furchgott RF: Endothelium-derived relaxing factor: discovery, early studies, and identification as nitric oxide. Biosci Rep 1999;19: 235-251.

10 Karim SM, Rhee AY, Given AM, Faulx MD Hoit BD, Brozovich FV: Vascular reactivity in heart failure. Role of myosin light chain phosphatase. Circ Res 2004;95:612-619.

$\checkmark 11$ Chen FC, Ogut O, Rhee AY, Hoit BD, Brozovich FV: Captopril prevents myosin light chain phosphatase isoform switching to preserve normal cGMP-mediated vasodilatation. J Mol Cell Cardiol 2006;41:488-495.

12 Ararat E, Brozovich FV: Losartan decreases p42/44 MAPK signaling and preserves LZ+ MYPT1 expression. PLoS One 2009;4:e5144.

13 Huang QQ, Fisher SA, Brozovich FV: Unzipping the role of myosin light chain phosphatase in smooth muscle cell relaxation. J Biol Chem 2004;279:597-603. 


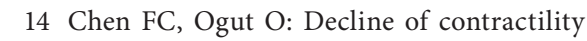
during ischemia-reperfusion injury: actin glutathionylation and its effect on allosteric interaction with tropomyosin. Am J Physiol Cell Physiol 2006;290:C719-C727.

15 Han YS, Ogut O: Regulation of fibre contraction in a rat model of myocardial ischemia. PLoS One 2010;5:e9528.

16 Christopher B, Pizarro GO, Nicholson B, Yuen $S$, Hoit BD, Ogut O: Reduced force production during low blood flow to the heart correlates with altered troponin I phosphorylation. J Muscle Res Cell Motil 2009;30:111-123.

17 Mulvany MJ, Halpern W: Contractile properties of small arterial resistance vessels in spontaneously hypertensive and normotensive rats. Circ Res 1977;41:19-26.

18 Mulvany MJ, Nilsson H, Flatman JA: Role of membrane potential in the response of rat small mesenteric arteries to exogenous noradrenaline stimulation. J Physiol 1982;332: 363-373.

-19 Mulvany MJ, Nilsson H, Flatman JA, Korsgaard N: Potentiating and depressive effects of ouabain and potassium-free solutions on rat mesenteric resistance vessels. Circ Res 1982;51:514-524.

20 Yuen SL, Ogut O, Brozovich FV: Nonmuscle myosin is regulated during smooth muscle contraction. Am J Physiol 2009;297:H191H199.

-21 Richards CT, Ogut O, Brozovich FV: Agonist-induced force enhancement: the role of isoforms and phosphorylation of the myosin-targeting subunit of myosin light chain phosphatase. J Biol Chem 2002;277:44224427.

22 Golomb E, Ma X, Jana SS, Preston YA, Kawamoto S, Shoham NG: Identification and characterization of nonmuscle myosin II-C, a new member of the myosin II family. J Biol Chem 2004;279:2800-2808.

-23 Phillips CL, Yamakawa K, Adelstein RS: Cloning of the cDNA encoding human nonmuscle myosin heavy chain-B and analysis of human tissues with isoform-specific antibodies. J Muscle Res Cell Motil 1995; 16:379_ 389.

24 Wang F, Kovacs M, Hu A, Limouze J, Harvey EV, Sellers JR: Kinetic mechanism of nonmuscle myosin IIB: functional adaptations for tension generation and maintenance. J Biol Chem 2003;278:27439-27448.

25 Kovacs M, Wang F, Hu A, Zhang Y, Sellers JR: Functional divergence of human cytoplasmic myosin II: kinetic characterization of the non-muscle IIA isoform. J Biol Chem 2003;278:38132-38140.

26 Rhee AY, Ogut O, Brozovich FV: Nonmuscle myosin, force maintenance, and the tonic contractile phenotype in smooth muscle. Pflugers Arch 2006;452:766-774.
27 Limouze J, Straight AF, Mitchison T, Sellers JR: Specificity of blebbistatin, an inhibitor of myosin II. J Muscle Res Cell Motil 2004;25: 337-341.

28 El-Yazbi AF, Johnson RP, Walsh EJ, Takeya K, Walsh MP, Cole WC: Pressure-dependent contribution of Rho kinase-mediated calcium sensitization in serotonin-evoked vasoconstriction of rat cerebral arteries. J Physiol 2010;588:1747-1762.

29 Walsh MP, Thornbury K, Cole WC, Sergeant G, Hollywood M, McHale N: Rho-associated kinase plays a role in rabbit urethral smooth muscle contraction, but not via enhanced myosin light chain phosphorylation. Am J Physiol Renal Physiol 2011;300:F73-F85.

30 Alioua A, Tanaka Y, Wallner M, Hofmann F, Ruth P, Meera P: The large conductance, voltage-dependent, and calcium-sensitive $\mathrm{K}+$ channel, Hslo, is a target of cGMP-dependent protein kinase phosphorylation in vivo. J Biol Chem 1998;273:32950-32956.

31 Schmidt HH, Lohmann SM, Walter U: The nitric oxide and cGMP signal transduction system: regulation and mechanism of action. Biochem Biophys Acta 1993;1173:153175.

32 Fukao M, Mason HS, Britton FC, Kenyon JL, Horowitz B, Keef KD: Cyclic GMP-dependent protein kinase activates cloned $\mathrm{BKCa}$ channels expressed in mammalian cells by direct phosphorylation at serine 1072. J Biol Chem 1999;274:10927-10935.

33 Dou D, Ma H, Zheng X, Ying L, Guo Y, Yu X, Gao Y: Degradation of leucine zipper-positive isoform of MYPT1 may contribute to development of nitrate tolerance. Cardiovasc Res 2010;86:151-159.

- $34 \mathrm{Ma} \mathrm{H}, \mathrm{He} \mathrm{Q}$, Dou D, Zheng X, Ying L, Wu Y, Raj JU, Gao Y: Increased degradation of MYPT1 contributes to the development of tolerance to nitric oxide in porcine pulmonary artery. Am J Physiol Lung Cell Mol Physiol 2010;299:L117-L123.

35 Ogut O, Brozovich FV: The potential role of MLC phosphatase and MAPK signalling in the pathogenesis of vascular dysfunction in heart failure. J Cell Mol Med 2008;12:21582164.

36 Kim HR, Appel S, Vetterkind S, Gangopadhyay SS, Morgan KG: Smooth muscle signalling pathways in health and disease. J Cell Mol Med 2008;12:2165-2180.

37 Aburto TK, Lajoie C, Morgan KG: Mechanisms of signal transduction during alpha 2-adrenergic receptor-mediated contraction of vascular smooth muscle. Circ Res 1993;72: 778-785.

38 Suzuki K, Hata S, Kawabata Y, Sorimachi H: Structure, activation, and biology of calpain. Diabetes 2004;53(suppl 1):S12-S18.

-39 Coux O, Tanaka K, Goldberg AL: Structure and functions of the 20s and 26s proteasomes. Annu Rev Biochem 1996;65:801-847.
40 Zong C, Young GW, Wang Y, Lu H, Deng N, Drews O, Ping P: Two-dimensional electrophoresis-based characterization of posttranslational modifications of mammalian $20 \mathrm{~S}$ proteasome complexes. Proteomics 2008;8:5025-5037.

41 Drews O, Tsukamoto O, Liem D, Streicher J, Wang Y, Ping P: Differential regulation of proteasome function in isoproterenol-induced cardiac hypertrophy. Circ Res 2010; 107:1094-1101.

42 Kapadia MR, Eng JW, Jiang Q, Stoyanovsky DA, Kibbe MR: Nitric oxide regulates the $26 \mathrm{~S}$ proteasome in vascular smooth muscle cells. Nitric Oxide 2009;20:279-288.

-43 Zhang JC, Kim S, Helmke BP, Yu WW, Du KL, Lu MM, Strobeck M, Yu Q, Parmacek MS: Analysis of SM22alpha-deficient mice reveals unanticipated insights into smooth muscle cell differentiation and function. Mol Cell Biol 2001;21:1336-1344.

-44 Shapland C, Hsuan JJ, Totty NF, Lawson D: Purification and properties of transgelin: a transformation and shape change sensitive actin-gelling protein. J Cell Biol 1993;121: 1065-1073.

45 Ali F, Chin L, Pare PD, Seow CY: Mechanism of partial adaptation in airway smooth muscle after a step change in length. J Appl Physiol 2007;103:569-577.

-46 Murry CE, Jennings RB, Reimer KA: Preconditioning with ischemia: a delay of lethal cell injury in ischemic myocardium. Circulation 1986;74:1124-1136.

47 Bolli R, Marban E: Molecular and cellular mechanisms of myocardial stunning. Physiol Rev 1999;79:609-634.

-48 Freis ED, Schnaper HW, Johnson RL, Schreiner GE: Hemodynamic alterations in acute myocardial infarction. I. Cardiac output, mean arterial pressure, total peripheral resistance, central and total blood volumes, venous pressure and average circulation time. J Clin Invest 1952;31:131-140.

49 Khatri JJ, Joyce KM, Brozovich FV, Fisher SA: Role of myosin phosphatase isoforms in cGMP-mediated smooth muscle relaxation. J Biol Chem 2001;276:37250-37257.

50 Payne MC, Zhang HY, Prosdocimo T, Joyce KM, Koga Y, Ikebe M, Fisher SA: Myosin phosphatase isoform switching in vascular smooth muscle development. J Mol Cell Cardiol 2006; 40:274-282.

51 Payne MC, Zhang HY, Shirasawa Y, Koga Y, Ikebe M, Benoit JN, Fisher SA: Dynamic changes in expression of myosin phosphatase in a model of portal hypertension. Am J Physiol 2004;286:H1801-H1810.

$52 \mathrm{Lu}$ Y, Zhang H, Gokina N, Mandala M, Sato O, Ikebe M, Osol G, Fisher SA: Uterine artery myosin phosphatase isoform switching and increased sensitivity to SNP in a rat L-NAME model of hypertension of pregnancy. Am J Physiol Cell Physiol 2008;294:C564-C571. 